\title{
Urinary Urgency
}

National Cancer Institute

\section{Source}

National Cancer Institute. Urinary Urgency. NCI Thesaurus. Code C50792.

An acute and compelling urge to urinate. 